\title{
Dispositivo automatizado para control de micro corrosión generada en convertidor de ozono instalado en área de producción de industria aeroespacial de Mexicali
}

\section{Automated device for micro-corrosion control generated in ozone converter installed in Mexicali's aerospace industry production}

ROMERO-SAMANIEGO, Elizabeth ${ }^{1} \dagger^{*}$, TOLEDO-PEREA, Sandra Luz ${ }^{1}$, LOPEZ-RODRIGUEZ, Rogelio $^{1}$ y LÓPEZ-BADILLA, Gustavo ${ }^{1}$

\author{
${ }^{I}$ Tecnológico Nacional de México/I. T. de Ensenada, Ensenada, Baja California, México. \\ ${ }^{2}$ Universidad UNEA, Mexicali, Baja California, México.
}

ID $1^{\mathrm{er}}$ Autor: Elizabeth, Romero-Samaniego / ORC ID: 0000-0002-9290-0879, CVU CONACYT ID: 499629

ID $1^{\text {er }}$ Coautor: Sandra Luz, Toledo-Perea / ORC ID: 0000-0001-6345-2125, CVU CONACYT ID: 27764

ID $2^{\text {do }}$ Coautor: Rogelio, Lopez-Rodriguez / ORC ID: 0000-0002-8857-9023, CVU CONACYT ID: 121516

ID $3^{\text {er }}$ Coautor: Gustavo, López-Badilla / ORC ID: 0000-0001-5302-6210, CVU CONACYT ID: 741919

DOI: $10.35429 /$ JTIP.2019.9.3.24.32

Recibido 23 de Octubre, 2019; Aceptado 04 Diciembre, 2019

\begin{abstract}
Resumen
Debido a fallas en convertidores de ozono usados en la industria aeroespacial y manufacturados en una empresa ubicada en la ciudad de Mexicali, y que generaba un problema de salud en interiores de aeronaves comerciales por mal funcionamiento de los convertidores de ozono, se evaluó el ambiente interno de la compañía mencionada y localizada en zona desértica del noroeste de la República Mexicana. El análisis se llevó a cabo con equipos especializados y un dispositivo de detección y control de sustancias químicas derivadas del azufre y depositadas en superficies metálicas de conexiones eléctricas de los convertidores de ozono instalados en una sección de las cabinas de aeronaves. Con el equipo de monitoreo, se detectó rápidamente el ambiente corrosivo en los interiores de la empresa y con el desarrollo de un dispositivo de detección de fallas eléctricas en los convertidores de ozono, disminuyeron las fallas en $70 \%$. Se realizaron microanálisis con la técnica de Microscopia de Barrido Electrónico (MBE), para identificar las sustancias contaminantes depositadas en las superficies metálicas de conexiones eléctricas de los convertidores de ozono, así como los niveles de concentración de dichas sustancias. El dispositivo automatizado para el control de la micro corrosión fue diseñado, fabricado y puesta en prueba, para detectar los periodos de deposición de las sustancias químicas derivadas del azufre. Además, se instalaron filtros especializados para disminuir el ingreso a los interiores de la empresa en Mexicali, y con ello tener un ambiente regulado y así evitar la generación de la micro corrosión. El estudio se realizó del 2017 al 2018.
\end{abstract}

Procesos industriales, Micro corrosión, Ambiente industrial corrosivo

\begin{abstract}
Due to failures in ozone converters used in the aerospace industry and manufactured in a company located in the city of Mexicali, and that generated a health problem in commercial aircraft interiors due to the malfunction of ozone converters, the internal environment was evaluated of the company mentioned and located in the desert area of the northwest of the Mexican Republic. The analysis was carried out with specialized equipment and a device for detecting and controlling chemical substances derived from sulfur and deposited on metallic surfaces of electrical connections of ozone converters installed in a section of aircraft cabins. With the monitoring equipment, the corrosive environment in the interiors of the company was quickly detected and with the development of an electrical fault detection device in the ozone converters, the failures were reduced by $70 \%$. Microanalysis was performed using the Electronic Scanning Microscopy (MBE) technique, to identify the contaminating substances deposited on the metallic surfaces of the electrical connections of the ozone converters, as well as the concentration levels of said substances. The automated device for micro corrosion control was designed, manufactured and tested, to detect periods of deposition of sulfur-derived chemicals. In addition, specialized filters were installed to reduce the entrance to the interiors of the company in Mexicali, and thus have a regulated environment and thus avoid the generation of micro corrosion. The study was conducted from 2017 to 2018.
\end{abstract}

Industrial processes, Micro corrosion, Corrosive industrial environment

Citación: ROMERO-SAMANIEGO, Elizabeth, TOLEDO-PEREA, Sandra Luz, LOPEZ-RODRIGUEZ, Rogelio y LÓPEZBADILLA, Gustavo. Dispositivo automatizado para control de micro corrosión generada en convertidor de ozono instalado en área de producción de industria aeroespacial de Mexicali. Revista de Tecnologías en Procesos Industriales. 2019 3-9: 24 32

\footnotetext{
* Correspondencia del Autor (Correo electrónico: eromero@ite.edu.mx)

$\dagger$ Investigador contribuyendo como primer autor.
} 


\section{Introducción}

La presencia de malos olores y ambientes que deben ser desinfectados en los interiores de aeronaves, es una preocupación para las autoridades de las empresas de aviación y de gobierno que son las que impulsan nuevas leyes en las actividades de tráfico de vuelos. Una gran cantidad de personas utilizan los traslados en aeronaves y son expuestos a este tipo de ambientes, siendo en ocasiones propensos a sufrir de algún tipo de padecimiento en los vuelos y posteriormente ser hospitalizados. Esto puede originar la posibilidad de que los pasajeros demanden a las compañías de aviación, generando una preocupación a los dueños y personal de este tipo de empresas ${ }^{1}$. Es por esto que se han dedicado a evitar la presencia de ambientes infectados, instalando en los aviones convertidores de ozono, que contienen dispositivos electrónicos que generan el proceso químico de desinfección de los ambientes interiores de las aeronaves.

Solo que en ocasiones los convertidores de ozono, no operan adecuadamente, debido a que se presenta una micro corrosión en las terminales de sus componentes electrónicos que realizan las funciones de conversión. En ocasiones una persona con problemas cardiovasculares en un vuelo, puede sufrir de un grave problema cardiovascular y pudiera fallecer en pleno vuelo, por los ambientes infectados, debido a veces a la falta de oxígeno limpio, y pudiendo ocurrir un fallecimiento. $\mathrm{Si}$ esto ocurriera principalmente en la mayor parte de los países del mundo, podría ser un grave suceso que generara una demanda legal, que podría tener serias consecuencias a las compañías de aviación involucradas en este tipo de sucesos. El gran crecimiento comercial de la industria de la aviación, ha propiciado el desarrollo de nuevas tecnologías en el control de las funciones de las aeronaves y con ello un mejor control en los procesos de fabricación que cada día son más complejos y utilizan con mayor frecuencia dispositivos electrónicos a nivel micro y nano escala $^{2}$.

Estos componentes electrónicos están propensos a sufrir de un deterioro en sus terminales eléctricas por la falta de control en los ambientes internos de las empresas de fabricación de sistemas de aviación.
El deterioro generado es por un ambiente corrosivo $^{3}$, y esto causa un mal funcionamiento de los sistemas de aviación que son inspeccionados minuciosamente por elaborar operaciones de gran importancia que no deben de generar fallas para evitar algún suceso trágico en las actividades de aviación comercial.

\section{La industria de la aviación}

Este tipo de industria ha crecido enormemente en los últimos diez años, y se ha transformado muy rápidamente por los avances tecnológicos que se han aplicado tanto a los procesos de fabricación, como a los sistemas que operan las aeronaves. Cada vez más los sistemas utilizados en aeronaves son más pequeños porque deben ser implementados nuevos dispositivos ${ }^{4}$ para nuevas funciones que optimicen la operación de las actividades en los vuelos comerciales.

La mayor parte de las empresas de aviación principalmente de Estados Unidos, desarrollan sus procesos de ensamble final en este país, y otras operaciones son elaboradas en otros países, siendo México uno de estos, donde se instalan empresas subsidiarias por la cercanía de las empresas matriz y un menor costo de mano de obra.

Una de las ciudades donde están instaladas algunas empresas que desarrollan algunos sistemas de las aeronaves, es Mexicali, donde alrededor del $50 \%$ pertenecen a productos y subproductos de este tipo de industria. Esta ciudad tiene la ventaja de estar ubicada en la zona fronteriza con California, donde se tiene una gran infraestructura terrestre en este estado de Estados Unidos, y tiene una gran diversidad de rutas hacia aeropuertos y puertos marítimos, por donde se envían los productos y subproductos de la industria aeronáutica y están cerca de Mexicali ${ }^{5}$.

Una de las desventajas de esta ciudad ubicada en una zona desértica es la gran preocupación de autoridades gubernamentales de un tema de interés social y de salud, como lo es la contaminación atmosférica que genera danos a la salud de la población de Mexicali y deterioro a los materiales, esencialmente de tipo metálico, siendo dos de los que son más afectados como el cobre y estaño, utilizados en terminales eléctricas de los productos y subproductos de la industria aeronáutica. 
Uno de los efectos que genera un daño a los materiales mencionados, es la desintegración de vías de conductividad eléctrica que unen a los micro dispositivos como se muestra en la figura 1 , que generan falta de conductividad eléctrica en ciertos micro componentes electrónicos y con ello una mala funcionalidad que causa una operación defectuosa de algún sistema usado en las aeronaves. Estas fallas de los sistemas defectuosos, pueden causar graves sucesos, como lo es el caso de la falta de ambientes desinfectados, pudiendo originar un síntoma grave de salud a algún pasajero con problemas cardiovasculares o de otro tipo de salud.

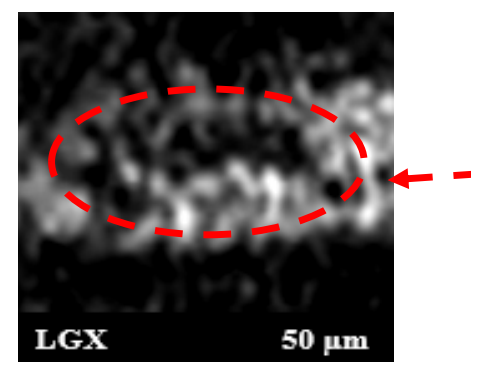

Soldadura de
estaño deteriorada
por el efecto de la
corrosión en vía de
conducción
eléctrica de micro

Figura 1 Vía de conductividad eléctrica desintegrada que une micro dispositivos electrónicos

Fuente. Microanálisis del estudio en empresa aeroespacial de Mexicali

La vía de conductividad mostrada en la figura 1, representa una conexión eléctrica dañada de un convertidor de ozono fabricado en una industria de aeronáutica ubicada en la ciudad de Mexicali, donde los niveles estándares de contaminación del aire sobrepasan en los exteriores de las industrias y penetran a los interiores de las empresas, generando ambientes agresivos a las superficies metálicas de las terminales de conexiones de los micro dispositivos electrónicos ${ }^{6}$. La corrosión presentada en el convertidor de ozono utilizado en la aviación de la figura 1, mostró fallas de conductividad eléctrica y con ello fue considerado un producto defectuoso que fue necesario elaborar un retrabajo y con ello un gasto innecesario representando perdidas económicas a la empresa donde se fabrican este tipo de sistemas de aeronaves.

\section{Corrosión atmosférica}

Es un tipo de deterioro de superficies metálicas expuestas a ambientes corrosivos donde en los interiores causa un mayor daño a los metales por no generar un intercambio de aireación y con ello tener un ambiente que provoca severos daños principalmente a las conexiones eléctricas de los dispositivos electrónicos ${ }^{7}$.
La figura 1 representada en la sección anterior, es un ejemplo de lo que ocurre por la corrosión atmosférica y si un sistema es usado en especial en las aeronaves, puede operar inadecuadamente y en operaciones de riesgo como en despegues, aterrizajes o en pleno vuelo de aeronaves; puede ser originar accidentes trágicos. Es por esto el control de los interiores de la industria aeroespacial respecto a mantener ambientes limpios y evitar con ello la generación de corrosión a nivel macro, micro o nano escala. Estudios desarrollados con anterioridad por Veleva y colaboradores, y López y colaboradores, indican que la ciudad de Mexicali es una de las ciudades más contaminadas de la República Mexicana, siendo esto un factor negativo para las empresas que utilizan macro, micro y nano componentes electrónicos ${ }^{1,2}$.

\section{La industria aeroespacial en el noroeste de la República Mexicana}

La zona donde está ubicada la ciudad de Mexicali es de gran importancia en el desarrollo socioeconómico de la República Mexicana, donde se tiene una gran infinidad de empresas principalmente del ramo electrónico, aeroespacial, metalmecánico, médico, textiles, cartón y plásticos, donde también se ubican las ciudades de Tijuana y Ensenada. Estas tres ciudades son las de mayor importancia en el estado de Baja California, que ha desarrollado clúster del ramo electrónico y aeroespacial, sin tener un éxito del $100 \%$, por no considerar algunos factores como lo es la presencia de corrosión, que aun teniendo expertos en esta área no han podido control totalmente este fenómeno de tipo electroquímico ${ }^{8,9}$.

La presencia de corrosión atmosférica en los interiores de la industria aeroespacial esencialmente de esta región considerada como zona desértica, ha ido en incremento aun cuando es en pequeños porcentajes, ocasionando enormes pérdidas económicas a las empresas subsidiarias de las grandes matrices ubicadas en Estados Unidos.

Según reportes de estudios anteriores, se han incrementado los índices de productos defectuosos por la presencia de la corrosión, hasta de un $20 \%$, que se ocasiona pérdidas millonarias a las compañías, que algunas de estas han considerado cambiar sus empresas subsidiarias a otras regiones del país, para aprovechar el bajo costo de la mano de obra en México.

ROMERO-SAMANIEGO, Elizabeth, TOLEDO-PEREA, Sandra Luz, LOPEZ-RODRIGUEZ, Rogelio y LÓPEZ-BADILLA, Gustavo. Dispositivo automatizado para control de micro corrosión generada en convertidor de ozono instalado en área de producción de industria aeroespacial de Mexicali. Revista de Tecnologías en Procesos Industriales. 2019 


\section{Convertidor de ozono}

Es un sistema que tiene la función de producir ozono con tres átomos de oxigeno por medio de la actividad con alta tensión eléctrica del fenómeno llamado "Efecto Corona"10. Este proceso es utilizado en interiores de ambientes para eliminar malos olores y lograr la desinfección del aire, donde personas permanecen por algunos periodos de tiempo, como lo son los traslados en aeronaves. Además, es usado en centros de salud y hospitales para evitar un contagio mayor de los pacientes.

Este sistema debe operar adecuadamente en las aeronaves para evitar contagios de personas propensas a sufrir algún padecimiento de salud, principalmente de tipo respiratorio.

\section{Ambientes infectados en aeronaves}

En los traslados de personas por aviación, se pueden generar ambientes infecciosos por la presencia de personas enfermas en vuelos comerciales y si no se tiene un adecuado proceso de intercambio de aireación con convertidores de ozono, se pueden contagiar otros pasajeros.

Además, en los procesos de alimentación en los vuelos comerciales, se presentan desechos alimenticios y en ocasiones la falta de higiene en sanitarios, ocasiona ambientes infecciosos, que pueden afectar a ciertos pasajeros propensos a enfermedades respiratorias que conlleven a síntomas más graves como efectos en el sistema cardiovascular ${ }^{10}$.

\section{Metodología del estudio}

El estudio se realizó en cuatro vertientes, donde se obtuvo información relevante que fue proporcionada a la empresa en la cual se elaboró la investigación.

Las cuatro etapas del estudio se muestran a continuación, con lo cual se mejoró el ambiente de interiores y con ello la reducción de porcentajes de fallas de los convertidores de ozono fabricados en esta región noroeste de la República Mexicana. Se utilizaron los estándares

$\operatorname{ASTM}^{11,12,13}$ e ISO $^{14,15}$. Enseguida se explican las cuatro etapas desarrolladas en la investigación: a)

Análisis del ambiente en interiores. Se desarrolló para evaluar la presencia de partículas contaminantes, en esencial las derivadas del azufre, nitrógeno y carbono provenientes del exterior de la empresa evaluada. Las principales fuentes de contaminación de estas partículas fueron los vehículos automotores, donde en la ciudad de Mexicali se tienen en base a estudios de expertos de esta área, en promedio 2 autos por familia, siendo una de las ciudades más contaminadas de México y de Latinoamérica. La evaluación se llevó a cabo con el monitor de calidad del aire para interiores B06XG6XLX7 marca Instrukart, como equipo especializado.

b) Evaluación de fallas de convertidores de ozono. Se elaboró para determinar la causa principal de las fallas eléctricas, que generaban productos defectuosos y no operaban adecuadamente. Esto generaba, en los procesos de pruebas de simulación, que los convertidores de ozono no desinfectarán ambientes infectados que son similares a los presentados en los vuelos comerciales y con ello generar pérdidas en los procesos de fabricación, que preocupaba en gran medida a gerentes y personal especializado de la empresa.

c) Uso de técnica MBE. Se realizó para determinar los tipos de partículas contaminantes y su nivel de concentración que generaba el deterioro de las superficies metálicas de las terminales eléctricas de los convertidores de ozono que originaba que fueran productos defectuosos.

d) Diseño y prueba de dispositivo de detección de fallas eléctricas en convertidores de ozono. Se desarrolló un dispositivo automatizado para detectar cuando sustancias contaminantes se adherían a las superficies metálicas de las terminales eléctricas dañadas de los convertidores de ozono, y con ello generaban variación de corriente y voltaje eléctrico, que generaba una señal de mala conductividad y con ello la indicación de que el convertidor de ozono evaluado era defectuoso y era necesario elaborar el retrabajo de éste. 
Con este sistema se detectaron más del $50 \%$ de convertidores de ozono defectuosos, que apoyó en gran medida a detectar antes los convertidores defectuosos antes de ser enviados a la matriz en una ciudad de Estados Unidos. El dispositivo diseñado y puesto a prueba se representa enseguida como un sistema de bloques.

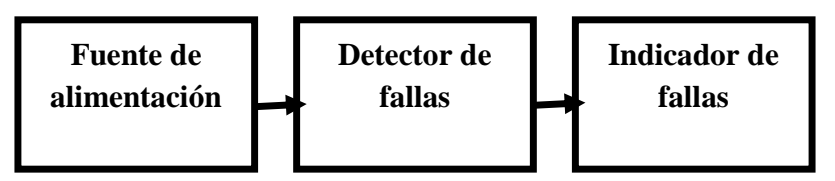

Figura 2 Etapas de operación del dispositivo detector de fallas eléctricas en convertidores de ozono

Fuente: Diseño y prueba por investigadores participantes

El dispositivo funciona con una fuente de alimentación (a) de energía eléctrica para su operación, continuando con una etapa de detección de fallas eléctricas (b), con dos cables eléctricos conectados a las terminales de los convertidores de ozono evaluando los niveles de conductividad eléctrica (corriente y voltaje eléctrico en el orden de microamperios) y por último se tiene el indicador (c) que muestra cuando la intensidad de corriente y voltaje no son adecuados para la operación adecuada del convertidor de ozono. En la figura 3a, se muestra solo el circuito electrónico que genera el funcionamiento de la detección de fallas, siendo operado por dos transistores 2N2222, dos resistores de $1 \mathrm{~K}-\mathrm{Omh}$ y un relevador de 12 voltios de corriente directa a 120 voltios de corriente alterna para ser conectado a un indicador con un foco de 120 voltios y 60 watts, muy utilizado en actividades industriales y hasta en domésticas. Estos dispositivos son conectados en la entrada del sistema de detección de fallas por una fuente de alimentación de 12 voltios de corriente detecta. En la figura 3b, se observa la parte inferior del circuito electrónico con las conexiones unidas con material de estaño, que es un gran conductor eléctrico.

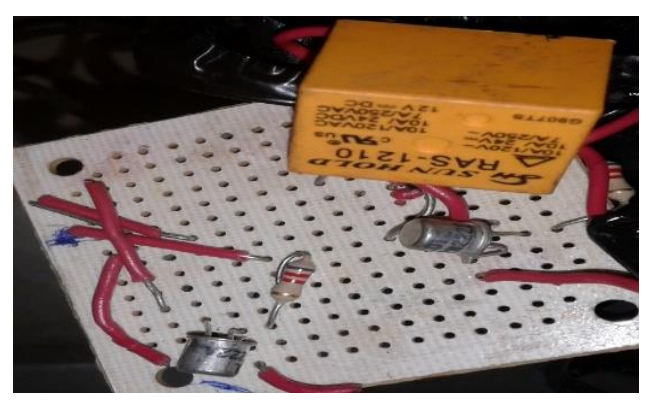

Figura 3a Vista frontal del circuito electrónico detector de fallas eléctricas de los convertidores de ozono.

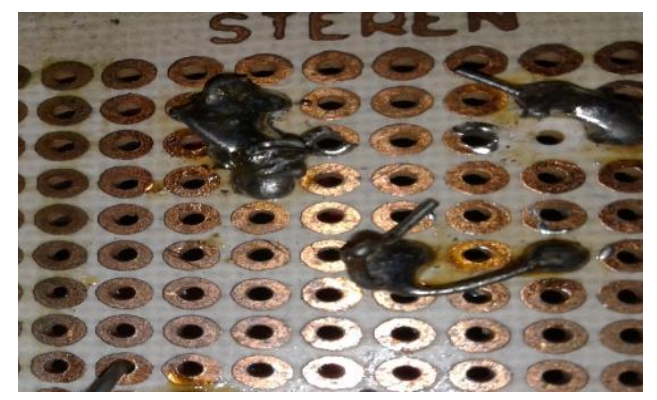

Figura 3a Vista inferior del circuito electrónico detector de fallas eléctricas de los convertidores de ozono

El análisis fue elaborado con un proceso con ecuaciones diferenciales, siguiendo la siguiente representación matemática:

$\frac{d y}{d x}=f(x, y)$

Como series finitas, elaborado por Issac Newton en $1671^{16}$.

\section{Resultados}

La investigación desarrollada reveló información importante en el proceso de fabricación de convertidores de ozono y de interés para otro tipo de industrias que utilizan dispositivos electrónicos a nivel micro y macro en sistemas de diversos productos fabricados en la ciudad de Mexicali que es una zona desértica. Esta región noroeste de la República Mexicana, es considerada una zona donde los niveles de contaminación del aire en especial las partículas derivadas del azufre, nitrógeno, carbono y ozono, sobrepasan los estándares de calidad del aire, que generan daños tanto a la población como a los materiales en especial los de tipo metálico. Las autoridades de esta región han elaborados grandes esfuerzos sin lograr un control total y al ser una ciudad fronteriza con Estados Unidos, las autoridades del país del norte de México, proceden a reclamos de manera frecuente. La información del estudio se revela a continuación con los análisis respectivos de cada etapa elaborada.

\section{Correlación de ambientes interiores y fallas eléctricas}

El análisis de fallas eléctricas relacionado a factores climáticos en especial de temperatura $\left(^{\circ}\right.$ C) y humedad relativa (\%), genero información relevante que fue proporcionada a la empresa para lograr el control de ambientes de interiores con condiciones adecuadas para fabricar convertidores de ozono con buena funcionalidad.

ROMERO-SAMANIEGO, Elizabeth, TOLEDO-PEREA, Sandra Luz, LOPEZ-RODRIGUEZ, Rogelio y LÓPEZ-BADILLA, Gustavo. Dispositivo automatizado para control de micro corrosión generada en convertidor de ozono instalado en área de producción de industria convertidor de ozono instalado en área de producción de industria
aeroespacial de Mexicali. Revista de Tecnologías en Procesos Industriales. 2019 
La figura 3 muestra una parte del estudio de la relación de las variables mencionadas.

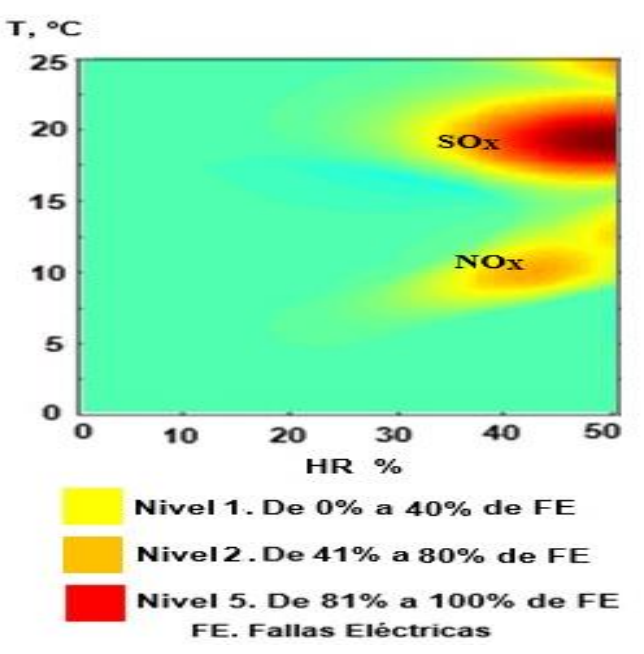

Figura 3 Correlación de factores climáticos, contaminación y fallas eléctricas (invierno del 2018)

En la figura anterior, se observa que a niveles de humedad entre el $30 \%$ al $50 \%$ y de 5 ${ }^{\circ} \mathrm{C}$ a $25{ }^{\circ} \mathrm{C}$, que fueron los que se presentaron con mayor frecuencia en la investigación. En estos rangos de estos factores climáticos, se presentaron niveles de $\mathrm{SO}_{\mathrm{X}}$ y $\mathrm{NO}_{\mathrm{X}}$, en la época de invierno, que fue el periodo del año en el cual sobrepasaron en mayor escala los niveles estándares en la ciudad de Mexicali, reglamentados por la Secretaria de Medio Ambiente de México con conjunto con la Environmental Protection Agency (EPA) de Estados Unidos. En el periodo de verano también se sobrepasaron los niveles estándares, pero en menor frecuencia y de igual forma se genera un daño a las conexiones eléctricas de los convertidores de ozono fabricados en la empresa instalada en esta ciudad del noroeste dela República Mexicana. Los factores climáticos evaluados en el estudio, en conjunto con la presencia de partículas contaminantes derivadas de azufre y nitrógeno: provenientes del exterior de la compañía de la gran fuente vehicular estimada en unos 200,000 autos en Mexicali, donde se desarrolló la investigación, generaron el deterioro de las superficies metálicas, y con ello las fallas eléctricas en los convertidores de ozono, ocasionando una gran cantidad de productos defectuosos. Se observa, además de los diversos niveles de frecuencia de fallas eléctricas con los tres diferentes colores, siendo el de color amarillo el de menor nivel con un rango de $0 \%$ a $40 \%$, de la frecuencia de fallas eléctricas, seguido del color naranja del $41 \%$ al $80 \%$ y por último el color rojo con el nivel del $81 \%$ al $100 \%$.
En base a esto se determinó la intensidad de riesgo de la generación de ambientes agresivos en interiores puede ocasionar un grave deterioro a los dispositivos electrónicos a nivel macro, micro y nano, causan grandes pérdidas económicas y en ocasiones graves tragedias si estos dispositivos operan en condiciones inadecuadas y ser usadas en funciones como el caso de la aviación en situaciones de despegue, vuelo y aterrizaje, que ha ocasionado accidentes trágicos y se ha determinado que el fenómeno de la corrosión ha sido un factor.

\section{Evaluación matemática}

Se realizó una evaluación con ecuaciones diferenciales de las variables involucradas que es la corriente y voltaje del sistema electrónico detector de fallas, y de los agentes contaminantes que generan variación en los amperios y voltios, debido a que se depositan en las conexiones eléctricas y el sistema electrónico no opera a su máximo rendimiento. En base a la evaluación se obtuvo la siguiente información:

\begin{tabular}{|l|r|r|}
\multicolumn{1}{|c}{ Contaminantes } & \multicolumn{1}{c}{$\begin{array}{c}\text { Corriente, } \\
\text { Amp }\end{array}$} & \multicolumn{1}{c|}{$\begin{array}{c}\text { Voltaje, } \\
\text { voltios }\end{array}$} \\
\hline $\mathrm{SO}_{2}$ & 1.45 & 11.07 \\
\hline $\mathrm{NO}_{2}$ & 1.68 & 11.48 \\
\hline $\mathrm{CO}$ & 1.79 & 11.66 \\
\hline $\mathrm{O}_{3}$ & 1.88 & 11.73 \\
\hline
\end{tabular}

Tabla 1 Correlación de variables eléctricas ambientales

La corriente eléctrica y voltaje en operación adecuada del sistema electrónico es de 2 amperios y 12 voltios con una tolerancia del $10 \%$ ( \pm 0.10 Amperios $\mathrm{y} \pm 1.2$ voltios). En la tabla 1 , se observan que los niveles de corriente y voltaje, se reducen a intensidades menores a la tolerancia, por lo que se indica que este sistema electrónico conectado a los convertidores como de prueba, tienen el mismo proceso de operación eléctrica y esto indica que fallaban, debiendo revisarlos y ser retrabajados, generando tiempo extra y con ello falta de entrega a tiempo y originando perdidas económicas a la empresa.

\section{Microanálisis de superficies metálicas}

Esta parte del estudio fue elaborada para determinar con mayor exactitud los agentes que se adhirieron a las superficies metálicas y generaron la falta de conductividad eléctrica, y con ello los convertidores de ozono defectuosos. A simple vista, no se puede apreciar el deterioro por lo cual fue necesario, realizar el microanálisis.

ROMERO-SAMANIEGO, Elizabeth, TOLEDO-PEREA, Sandra Luz, LOPEZ-RODRIGUEZ, Rogelio y LÓPEZ-BADILLA, Gustavo. Dispositivo automatizado para control de micro corrosión generada en convertidor de ozono instalado en área de producción de industria aeroespacial de Mexicali. Revista de Tecnologías en Procesos Industriales. 2019 
El agente contaminante que ocasionó un mayor daño fueron los derivados del azufre, de muchos autos que circulan en diversos periodos de tiempo del día, y que por una mala situación la empresa evaluada está ubicada a un lado de un boulevard muy transitado y esto hace que los agentes derivados del azufre pudieran llegar más fácil a los procesos industriales de los interiores de la compañía donde se realizó la investigación.

Como se menciona, el deterioro fue principalmente en las conexiones de estaño que unen los macro, micro y nano componentes electrónicos, y con ello la falta de conductividad eléctrica y sus consecuencias mencionadas anteriormente. Las figuras 4 y 5 muestran conexiones de estaño dañado, y con las ramas de conexión fracturadas.

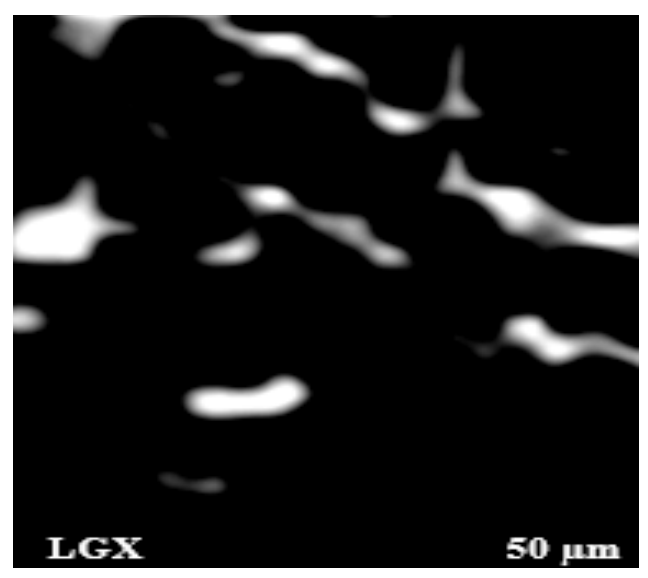

Figura 4 Microanálisis de soldadura de estaño dañada por partículas de $\mathrm{SO}_{\mathrm{X}}$ en verano.

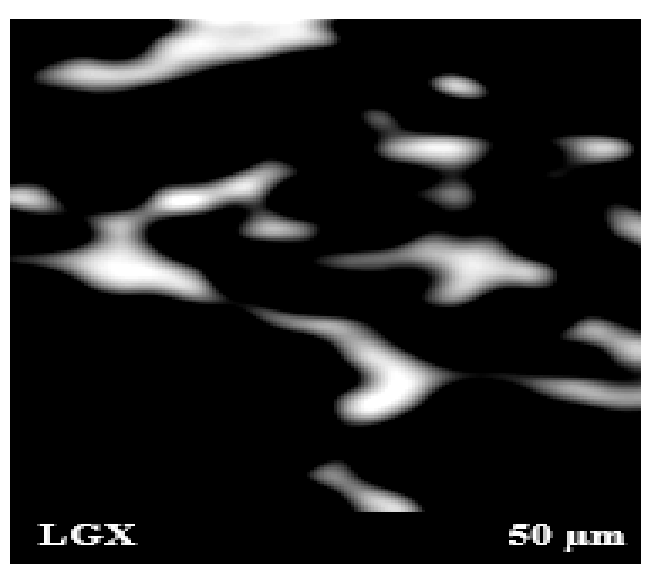

Figura 5 Microanálisis de soldadura de estaño dañada por partículas de $\mathrm{SO}_{2 \mathrm{x} e n}$ invierno (2018)

En la figura 4 se observa el microanálisis de la época de verano, donde se muestra las conexiones dañadas, pero con algunas pequeñas uniones, siendo un menor nivel de deterioro comparado con la figura 5 , donde se representa un daño mayor al observarse la figura con las conexiones totalmente desintegradas.
Esto ocasionó una mayor cantidad de convertidores de ozono defectuosos y la gran preocupación del personal de gerencia y especializado de la empresa evaluada.

\section{Evaluación de productos defectuosos}

Por la presencia de la corrosión en interiores de la compañía donde se elaboró el estudio, se desarrollaron análisis por periodos horarios, diarios, semanales, mensuales y estacionales del 2017 al 2018.

Por la gran cantidad de información se presenta en este texto, los datos más relevantes en las tablas 2 y 3 de la investigación que se explican a continuación.

\begin{tabular}{|c|c|c|c|c|c|}
\hline \multirow{2}{*}{$\begin{array}{l}\text { Factores } \\
\text { Semanas }\end{array}$} & \multirow[b]{2}{*}{$\mathrm{CPF}$} & \multicolumn{3}{|c|}{ ICTE \% } & \multirow{2}{*}{$\begin{array}{c}\text { ROEyMI, } \\
\%\end{array}$} \\
\hline & & CPD & $\begin{array}{c}\mathrm{MO} \\
\%\end{array}$ & $\begin{array}{c}\text { EyM, } \\
\%\end{array}$ & \\
\hline Octubre (1) & 5500 & 1082 & 26 & 24 & 56 \\
\hline Octubre (2) & 5000 & 1065 & 23 & 22 & 59 \\
\hline Octubre (3) & 4800 & 951 & 23 & 21 & 43 \\
\hline Octubre (4) & 4500 & 97 & 28 & 29 & 45 \\
\hline Noviembre (1) & 4300 & 943 & 26 & 27 & 48 \\
\hline Noviembre (2) & 4200 & 955 & 25 & 26 & 40 \\
\hline Noviembre (3) & 4100 & 959 & 26 & 27 & 43 \\
\hline Noviembre (4) & 4000 & 964 & 27 & 28 & 46 \\
\hline \multicolumn{6}{|c|}{$\begin{array}{l}\text { CPF. Cantidad fabricada por mes, CPD. Cantidad de productos } \\
\text { defectuosos por mes, ICTE (\%). Incremento de Costos por Tiempo Extra } \\
\text { (Porcentaje) - MO (Mano de Obra) - EyMI. (Equipo y Maquinaria } \\
\text { Industrial), ROEyMI (\%). Rendimiento Operativo de los Equipos y } \\
\text { Maquinaria Industrial (Porcentaje) - Cantidad de productos fabricados } \\
\text { contra fallas eléctricas y mecánicas, }\end{array}$} \\
\hline
\end{tabular}

Tabla 2 Análisis de corrosión atmosférica y productividad (Octubre y Noviembre del 2017) sin el dispositivo de detección de fallas eléctricas

La tabla 2 muestra los índices de productividad, donde se indica la cantidad total de producción en los meses de octubre que fue donde se generaron los mayores niveles de productos defectuosos en el 2017 cuando se inició el estudio.

Además, se observa la cantidad de productos defectuosos, así como el incremento del costo de los productos por los tiempos extra, relacionados a la operación de máquinas industriales y de la mano de obra.

También se representa, el rendimiento operativo como una correlación de la productividad con las maquinas industriales y la mano de obre contra los niveles de productos defectuosos. 
Los datos mostrados en la tabla 1 son parte del estudio sin haber aplicado el dispositivo de detección de fallas eléctricas en los convertidores de ozono usados en actividades de aviación, mostrando que a medida que transcurrían los días los índices de producción disminuían y en cambio se incrementan los niveles de los productos defectuosos, así como los costos por uso de tiempo extra y rendimiento operativo, que tenía bien preocupados al personal de gerencia y especializado. En la tabla 3 , se indica la misma información de la tabla, solo que los niveles de producción indican incrementos y disminución en los índices de productos defectuosos y del costo por tiempo extra y rendimiento operativo. Esto fue con el uso del dispositivo de detección de fallas eléctricas en los convertidores de ozono fabricados.

\begin{tabular}{|l|r|r|r|r|r|}
\hline Factores & CPF & CPD & \multicolumn{2}{c|}{ ICTE \% } & ROEyMI, \\
Semanas & TAM & TAM & $\begin{array}{c}\text { MO } \\
\text { EyM, }\end{array}$ & $\begin{array}{c}\text { Ro } \\
\%\end{array}$ \\
\hline Octubre (1) & 5000 & 248 & 8 & 7 & 73 \\
\hline Octubre (2) & 5300 & 243 & 7 & 9 & 74 \\
\hline Octubre (3) & 5500 & 237 & 7 & 7 & 76 \\
\hline Octubre (4) & 5700 & 234 & 6 & 6 & 76 \\
\hline Noviembre (1) & 5800 & 230 & 8 & 5 & 78 \\
\hline Noviembre (2) & 6300 & 227 & 9 & 4 & 80 \\
\hline Noviembre (3) & 6500 & 223 & 7 & 4 & 82 \\
\hline Noviembre (4) & 6700 & 220 & 5 & 3 & 83 \\
\hline CPF. Cantidad fabricada por mes, CPD. Cantidad de productos \\
defectuosos por mes, ICTE (\%). Incremento de Costos por Tiempo Extra \\
(Porcentaje) - MO (Mano de Obra) - EyMI. (Equipo y Maquinaria \\
Industrial), ROEyMI (\%). Rendimiento Operativo de los Equipos y \\
Maquinaria Industrial (Porcentaje) - Cantidad de productos fabricados \\
contra fallas eléctricas y mecánicas,
\end{tabular}

Tabla 3 Análisis de corrosión atmosférica y productividad (Octubre y Noviembre del 2018) con el dispositivo de detección de fallas eléctricas

En base a esto, se determinó que el dispositivo detector de fallas eléctricas, era necesario para incrementar los índices de productividad.

\section{Conclusiones}

El análisis desarrollado y el uso del dispositivo diseñado y puesta a prueba del sistema electrónico detector de fallas eléctricas en convertidores de ozono, fue de gran utilidad a la empresa que permitió elaborar la investigación. Existen diversos estudios anteriores de expertos en corrosión de una institución educativa de prestigio en la ciudad de Mexicali, pero no se había desarrollado un análisis tan detallado como éste elaborado, siendo de gran relevancia.
Esto es una gran aportación para la industria electrónica, aeroespacial, biomédica, electromecánica o similar donde se utilicen macro, micro y nano dispositivos electrónicos en equipos y maquinaria industrial y en productos fabricados en esta región de la República Mexicana. Esta zona es considerada por expertos ambientalistas como muy contaminada, y genera muchos problemas a la industria de esta región.

La corrosión atmosférica generada en los interiores de la empresa evaluada, fue por la presencia principalmente de los contaminantes derivados del azufre. Estos agentes contaminantes penetraron por rejillas, hendiduras y techo a la empresa donde se elaboró el estudio, y originó un ambiente agresivo. Los casos de ocurrencia de fallas eléctricas en los convertidores de ozono usados en aeronaves comerciales defectuosos, no generarían una adecuada desinfección del ambiente interior de las aeronaves. La presencia de corrosión originó la disminución de la productividad y calidad en la industria evaluada, y con ello pérdidas económicas, hasta perder un cliente por entrega tardía de los productos manufacturados. En base al microanálisis se determinó el tipo de corrosión observado, para la época de verano e invierno.

\section{Referencias}

1 L. Veleva, B. Valdez, G. Lopez, L. Vargas and J. Flores (2008) Atmospheric corrosion of electro-electronics metals in urban desert simulated indoor environment Corrosion Engineering Science and Technology, Vol. 43 No. 2 pp 149-155ISSN 148-422X.

2 Lopez, B.G., Valdez, S.B., Zlatev, K. R., Flores, P.J., Carrillo, B.M., Schorr, W. M. (2007) Corrosion of metals at indoor conditions in the electronics manufacturing industry Anti-Corrosion Methods and Materials Vol.54, Issue 6 pp 354-359.

3 López G., Tiznado H., Soto G., De la Cruz W., Valdez B., Schorr M., Zlatev R. (2010) Corrosión de dispositivos electrónicos por contaminación atmosférica en interiores de plantas de ambientes áridos y marinos Nova Scientia No. 5 Vol. 3(1) ISSN 2007-0705.

4 Moncmanova A. (2007) Environmental Deterioration of Materials, WIT Press Publishing ISBN 978-1-84564-032-3. 
5 Lopez B. G., Valdez S. B., Schorr W. M., Tiznado V. H., Soto H. G. (2010) Influence of climate factors on copper corrosion in electronic equipment's and devices AntiCorrosion Methods and Materials Vol. 57 Issue 3 pp 148-152.

6 Berresheim, H.; Wine, P.H. and Davies D.D. (1995). Sulfur in the Atmosphere. In Composition, Chemistry and Climate of the Atmosphere, ed. H.B. Singh. Van Nostran Rheingold ISBN.

7 M.F. Caggiano, E. Barkley, M. Sun, J.T. Kleban (2000). Electrical modeling of the chip scale ball grid array package at radio frequencies Original Research Microelectronics Journal, Volume 31, Issue 8, Pages 701-709.

8 M.F. Caggiano, R.M. Brush, J.T. Kleban, P.J. Chuaypradit (1998). Computer program that generates an electrical circuit model of the chip scale BGA Original Microelectronics Journal, Volume 29, Issue 12, December Pages 1013-1024

9 López Badilla Gustavo (2008) Caracterización de la corrosión en materiales metálicos de la industria electrónica en Mexicali, B.C Tesis de Doctorado Instituto de Ingeniería Universidad Autónoma de Baja California Mexicali, B.C. México.

10 Johnson E., Kelly R. Thomas D. (2012). Infected environments in commercial flights; Health and Environment Journal, Vol. 6 (3); pp. $45-56$.

11 ASTM G1 - 03 (2003) Standard Practice for Preparing, Cleaning, and Evaluating Corrosion Test Specimens

12 ASTM G4 - 01 (2008) Standard Guide for Conducting Corrosion Tests in Field Applications.

13 ASTM G31 - 72 (2004 Standard Practice for Laboratory Immersion Corrosion Testing of Metals.
14 ISO 11844-2 (2005) Corrosion of metals and alloys - Classification of low corrosivity of indoor atmospheres - Determination and estimation attack in indoor atmospheres. ISO, Geneva.

15 ISO 11844-1(2006) Corrosion of metals and alloys - Classification of low corrosivity of indoor atmospheres- Determination and estimation of indoor corrosivity. ISO, Geneva.

16 Newton, Isaac (1736) [1671]. Methodus Fluxionum et Serierum Infinitarum (The Method of Fluxions and Infinite Series) (en inglés) I. Opuscula. p. 66.
ROMERO-SAMANIEGO, Elizabeth, TOLEDO-PEREA, Sandra Luz, LOPEZ-RODRIGUEZ, Rogelio y LÓPEZ-BADILLA, Gustavo. Dispositivo automatizado para control de micro corrosión generada en convertidor de ozono instalado en área de producción de industria aeroespacial de Mexicali. Revista de Tecnologías en Procesos Industriales. 2019 